\title{
$\mathrm{T} 2$ 강조 복부자기공명영상에 대한 최적의 지방소거 기법의 정량적 평가 : TSE-SPIR 와 GE-PROSET 비교
}

구은회 ${ }^{1 *}$

${ }^{1}$ 청주대학교 방사선학과

\section{Quantitative Evaluation of Optimized Fat-Suppression Techniques for T2 Weighted Abdominal MR Imaging : Comparison of TSE-SPIR and GE-PROSET}

\author{
Eun-Hoe Goo $^{1^{*}}$ \\ ${ }^{1}$ Department of Radiological Science, Cheongju University
}

\begin{abstract}
요 약 본 실험의 목적은 TSE-SPIR 지방소거기법과 GE-PROSET 지방소거기법을 비교하여 복부자기공명영상에서 최적의 T2 강조 지방소거기법을 평가하고자 한다. 자기공명영상 검사는 16 채널 다중채널을 사용하여 $1.5 \mathrm{~T}(\mathrm{Philips}$, Medical System, Achieva)기기로 수행하였다. 모든 영상은 TSE-SPIR과 GE-PROSET을 이용하여 축면상(axial plane)을 얻었다. TSE-SPIR과 GE-PROSET에 대한 후복막 지방의 평균 SNRs는 $31.50,4.15$ 이었으며, 장간막 지방에 대한 SNRs는 32.39, 7.03이었다. TSE-SPIR과 GE-PROSET에서 장과 후복막 지방, 장간막 지방의 CNRs는 $152.69,74.54$ 와 $26.12,68.78$ 이었다. 장벽에 대한 묘출도는 TSE-SPIR(2.4), GE-PROSET(1.8)로 TSE-SPIR가 높게 나타났으며, 췌장벽 (pancreas wall)에 대한 묘출도는 TSE-SPIR(1.90), GE-PROSET(2.80)로 GE-PROSET가 높게 나타났다. 결론적으로, T2 강조 지방소거 복부자기공명영상에서 GE-PROSET 지방소거기법 보다 TSE-SPIR 지방소거기법이 우위성 있은 평가 를 얻었다.
\end{abstract}

\begin{abstract}
The purpose of this experiment is to evaluate of optimized FS techniques for T2 weighted abdominal MRI compared of TSE-SPIR fat suppression and GE-PROSET fat suppression. All MR examinations were performed on a $1.5 \mathrm{~T}$ (Philips, Medical System, Achieva) scanner using 16 channel mult-coils. All images were performed in the axial plane using TSE-SPIR and GE-PROSET. The mean SNRs of the retroperitoneal and mesenteric fat for TSE-SPIR and GE-PROSET were 31.50, 4.15 and 32.39, 7.03. The mean CNRs of the bowel and retroperitoneal, mesenteric fat for TSE-SPIR and GE-PROSET were 52.69, 74.54 and 26.12, 68.78). The delineation of bowel wall margins with TSE-SPIR(2.4) and GE-PROSET(1.8) were significantly improved using TSE-SPIR. The delineation of pancreas wall with TSE-SPIR(1.90), GE-PROSET(2.80) were significantly improved using GE-PROSET. In conclusion, TSE-SPIR fat suppression was superior to GE-PROSET fat suppression in T2 WI FS abdominal MRI.
\end{abstract}

Key Words : Contrast - to - Noise Ratio, Fat Suppression, Gradient Echo, Signal - to - Noise Ratio, Turbo Spin Echo

\section{1. 서론}

자기공명영상은(magnetic resonance imaging; MRI)은 물과 지방 사이에 화학적 이동현상을 가지고 있다. 지방 은 기본적으로 강한 신호를 가지고 있어 잘못된 인공물
을 형성하기도 한다. 지방소거(fat suppression)를 통해 영 상을 얻는데 가장 기본이 되는 방법으로 화학적 이동 선 택포화(chemical shift selective saturation; CHESS)기법이 다[1]. 이 기법은 화학적 이동(chemical shift)효과를 이용 하여 지방이나 물만을 선택적으로 영상화하는 기법으로

\footnotetext{
*Corresponding Author : Eun-Hoe Goo (Cheongju University)
}

Tel: +82-10-8750-5092 email: geh@cju.ac.kr

Received July 16, $2013 \quad$ Revised (1st August 5, 2013, 2nd September 3, 2013)

Accepted October 10, 2013 
서 물을 억제 시키거나 지방을 억제시켜 영상을 얻는 방 법이다. 이러한 원리를 이용하여 대부분 자기공명영상 검 사 시 지방소거 기법을 기본으로 모든 검사 영역에 포함 되어 있다. 특히 조영제 주입 전·후 복부(abdomen)[2] 및 사지(extremity)[3] 검사에서 기본으로 지방소거 기법 을 적용하여야만 진단이 가능하며, 그 외에 T2 강조 지방 소거기법은 급성 복부질환(acute abdomen disease)이 있 는 산모환자에게 유용한 가치가 있다고 한다[4]. 지방은 장(bowel), 췌장(pancreas), 신장(kidney) 주변에 많이 분 되어 있으며, 이러한 부위에 염증(inflammation), 전염 (infection), 또는 비정상적 유체물(abnormal fluid collections)의 원인으로 질환이 진행될 때 필수적으로 지 방소거 기법이 적용되어야만 평가가 가능하다[5-6]. 복 부 MRI는 호흡과 연관성이 깊으며, 또한 내부에 지방이 많이 분포 되어 있기 때문에 호흡정지 기법(breath- hold technique)을 적용하여 지방소거 영상을 획득하여야 만이 정확한 평가가 이루어진다. 복부 MRI에서 호흡정지 기법 을 적용하여 T2 강조 지방소거 펄스시퀀스는 half-Fourier acquisition single shot turbo spin echo(HASTE), turbo spin echo(TSE), gradient echo(GE)로 3 가지을 주로 적 용할 수가 있다[7]. HASTE 기법은 시간을 줄이기 위해 적은 수의 에코를 이용하는 방법이며 K-space의 에코 간 격은 일정하게 하면서 K-space의 일부만을 진 신호(true echo)로 얻고, 나머지는 거울데이터(mirrored data)로 채 우는 방법이다. 영상획득시간이 줄고 해상력 (resolution)이 좋은 특성이 있으나, 신호대 잡음비 (signal to noise ratio: SNR)가 감소하고, 흔들림이 생기는 단점 이 있다[8]. TSE 기법은 스핀에코 영상파형(spin echo sequence)을 고속영상법으로 변환시킨 방법이다. 검사소 요시간을 줄이기 위해 turbo factor를 사용하며, 유효에코 시간(effective TE)을 적용하여 K-space 중앙부분에 대조 도를 결정하게 하는 신호를 먼저 채우는 기법이다. 데이 터를 모으는 TE 시간이 서로 다르기 때문에 흔들림이 발 생할 수 있는 단점이 있으나, 고해상력과 대조도가 높은 영상을 얻을 수가 있다[9]. GE 기법은 짧은 TR(repetition to time), TE(echo to time)을 사용하며, 숙임각이 다른 HASTE, TSE에 비해 작기 때문에 검사시간이 짧은 기법 이나, $\mathrm{GE}$ 은 $\mathrm{RF}$ (radio frequence) 인가시간이 짧아 자장의 불균일성이 포함된 신호를 얻기 때문에 영상의 질(image quality)이 다소 떨어진다[10]. 본 실험에서 3종류의 기법 중 HASTE 기법은 복부 MRI 검사 시 사용하고 있지만, 이론적 장, 단점에 따라 기존 보고에 의하면 SNR과 CNR 이 TSE 비해 많이 떨어지기 때문에 평가를 하지는 않았 다[11]. 그러나 저자가 알기에 지방소거기법을 적용하여 $\mathrm{GE}$ 기법과 TSE 기법에 대한 우위성을 비교한 논문이 없
기 때문에 복부질환에 대한 최적의 정보를 제공하기 위 해 비교할 필요성이 있다고 생각한다. 또한 TSE 지방소 거기법은 $\mathrm{GE}$ 지방소거기법에 비해 검사 소요시간이 길 기 때문에 환자상태가 좋지 않은 경우에는 움직임이 있 는 영상보다 영상의 질은 떨어지지만 움직임이 없는 영 상이 환자에 대한 정보를 더욱더 많이 제공하기 때문에 $\mathrm{GE}$ 지방소거기법에 대한 대처가능성을 고려할 필요성이 있기 때문이다. 이에 본 실험의 목적은 $1.5 \mathrm{~T} \mathrm{MRI} \mathrm{기기를}$ 사용하여 복부 검사 시 지방소거기법으로 가장 많이 사 용되고 있는 TSE-SPIR과 GE-PROSET 기법을 비교하여 병변을 묘출하는데 가장 적합한 지방소거 기법을 알아보 고, 정량적 평가를 통하여 두 기법에 대한 임상적 유용성 을 고려하고자 한다.

\section{2. 대상 및 방법}

\section{1 대상}

2012년 10월부터 2013년 5월까지 총 30명(남자 : 15 명, 여자 15 명, 연령분포: $40 \sim 50$ 세(평균 $45.32 \pm 4.5$ 세) 의 정상인 지원자를 대상으로 하였으며, 모든 지원자는 사전에 MRI 검사 경험자만을 선정하여 실험을 하였다. 정량적인 데이터 분석을 위하여 병력 상에 폐질환 경험 이 있는 대상은 숨을 고르게 참지 못하기 때문에 모두 실 험대상에서 제외시켰다. 모든 환자는 검사를 받기 전 본 실험에 안전성에 관한 사전 동의서를 작성 후 검사를 하 였다.

\section{2 방법}

\subsection{1 자기공명영상 프로토콜(MRI protocol).}

본 실험에서 정량적 평가를 위해 선택한 장비는 1.5 Tesla 자기공명영상기기(Philips, Medical System, Achieva)를 선정하여 실험하였고, 데이터 수신을 위한 자기공명영상 코일로서 16 채널 다중코일을 사용하였다. 데이터 평가를 위해 영상획득은 축면상(axial plane)으로 하였으며, 적용 할 펄스시퀀스로는 TSE( TR : $2326 \mathrm{~ms}$, TE : $80 \mathrm{~ms}$, Matrix : 240x198, Slice thickness : 7mm, Gap : $1 \mathrm{~mm}$, FOV : $300 \mathrm{~mm}, \mathrm{FA}: 90^{\circ}$ )와 $\mathrm{GE}(\mathrm{TR}: 204 \mathrm{~ms}$, TE : 9ms, Matrix : 240x198, Slic thickness : 7mm, Gab 1mm, FOV : $300 \mathrm{~mm}, \mathrm{FA} 25^{\circ}$ )로 하였다. 이러한 매개변수의 조건은 검사 소요시간이 21 초, 17 초로 모두 호흡정지기법이 가 능하도록 설정을 하였다. 두 펄스 시퀀스에 대한 최적의 지방소거 기법을 알아보고, 평가하기 위하여 TSE에서는 SPIR 기법을 적용해 보았고, GE에서는 PROSET 기법을 
적용하였다.

\section{3 영상분석(Image analysis).}

\subsection{1 정량적 분석}

두 가지의 영상기법에서 후복막 지방과 장간막 지방에 $47.63 \mathrm{~mm}^{2}$ 로 관심부위(region of interest, 이하 ROI)를 설 정한 후 측정 하였다. 배경잡음의 신호강도는 위상부호화 방향(phase-encoding direction)인 복부표면의 전·후 측 공간에 $362.99 \mathrm{~mm}^{2}$ 로 환형의 관심 부위를 설정하였다. 이 기본 수치를 이용한 정량적 분석을 위해 사용한 지표 (parameter)는 신호대 잡음비, bowel 과 인접부위의 소거 된 지방에 대하여 대조도대 잡음비를 구하였다. 이들 parameter는 다음과 같이 정의한다.

1. SER: 후복막 지방과 장간막 지방의 ROI에서 얻은 평균 신호강도를 위상 부호화방향인 복부표면의 전 - 후 측 공간에서 설정한 ROI에서 얻은 배경잡음 신호강도의 표준편차로 나누었다. ROI의 크기는 같 았으며, 경계가 좋은 장의 중앙과 그 인접부위의 소 거(suppression)된 지방에 설정하였다. SNR을 평가 하는 방법으로 식 $(1,2)$ 과 같이 적용을 하였다[12].

$$
\begin{aligned}
& S N R_{1}=\frac{S I_{\text {retroperitoneal_fat }}}{S D_{\text {background noise }}} \\
& S N R_{2}=\frac{S I_{\text {mesenteric_fat }}}{S D_{\text {background noise }}}
\end{aligned}
$$

2. CNR: (장의 평균 신호강도에서 후복막 지방과 장간 막 지방의 관심영역에서 얻은 평균 신호강도를 잡 음의 표준편차의 형식으로 얻어졌다. $\mathrm{CNR}$ 을 평가 하는 방법으로 식 $(3,4)$ 과 같이 적용하였다[13].

$$
\begin{aligned}
C N R_{1}= & S I_{\text {bowel }}-S I_{\text {retroperitoneal_fat }} \\
& / S D_{\text {background noise }} \\
C N R_{2}= & S I_{\text {bowel }}-S I_{\text {mesenteric_fat }} \\
& / S D_{\text {background noise }}
\end{aligned}
$$

\subsection{2 정성적 분석}

두 가지의 MR기법으로 얻은 영상들은 각각 독립적으 로 두 명의 복부방사선과 의사와 국제 전문방사선사 1 명 에 의하여 평가되었다. 이들은 각 영상에 대하여 bowel wall margins의 묘출도( delineation)를 세 단계로 점수를 주었다. 구분의 단계는 불량(poor, 1), 보통(fair, 2), 양호 (good delineation, 3) 이었고 각 영상에 대하여 얻어진 점 수를 평균화 하여 비교하였다. 묘출도는 국소 신호강도가 미미하여 경계가 구분되지 않을 때 1점, 신호강도 변화가
미미하고, 경계가 뚜렷하지 않을 때 2점, 신호강도 변화 가 뚜렷하고, 경계가 좋을 때 3 점으로 평가하였다.

\section{4 통계분석}

두 가지 영상기법에서 정량적 분석으로 신호대 잡음비 와 대조도대 잡음비에 대한 통계적 유의성은 paired Student's t-test로 계산되었으며, 장벽(bowel wall)의 차이 (margins)에 대한 통계적 유의성은 Wilcoxon Rank test를 적용하였고, 통계에 사용된 프로그램은 SPSS 14.0을 이 용하여 $\mathrm{p}$ 값이 0.05 이하일 때 유의성을 두었다.

\section{3. 결과}

총 30 명의 정상인 지원자에서 정량적 분석결과 TSE-SPIR과 GE-PROSET기법을 적용했을 때 후복막 지 방의 평균 SNRs는 $31.50 \pm 05.98,4.15 \pm 00.98$ 이었으며 $(\mathrm{p}=0.00021)$, 장간막 지방의 SNRs는 $32.39 \pm 06.01,7.03$ \pm 0.2 .07 이었다 $(\mathrm{p}=0.00085)$. TSE-SPIR과 GE-PROSET에 서 장(bowel)과 후복막 지방, 장간막 지방의 CNRs는 $152.69 \pm 15.87,74.54 \pm 10.14(\mathrm{p}=0.00074)$ 와 $26.12 \pm$ $11.19,68.78 \pm 09.26(\mathrm{p}=0.00002)$ 이었다(Table 1). SNRs 비교에서는 TSE-SPIR 기법이 4배 이상의 아주 높은 값 을 얻어 많은 정보를 제공할 수 있을 것이며, CNRs 비교 에서도 TSE-SPIR 값이 2 배 이상의 높은 값을 얻어 지방 과 미세한 병변에 대한 차이를 뚜렷하게 하는 결과이다. 본 실험결과 얻은 영상을 이용하여 측정했을 때 장벽에 대한 묘출도는 TSE-SPIR(2.4 \pm 0.4$),$ GE-PROSET(1.8 \pm $0.3)$ 로 TSE-SPIR가 높게 나타났으며 $(\mathrm{p}=0.00027)$, 그림 1 에서 보여 주고 있다. 그림 1에서 a 그림(TSE-SPIR)이 b 그림(GE-PROSET)에 비해 원 (circle)을 보았을 때 소장 벽(small bowel wall)의 차이가 크게 구분되어 있으며, 장 과 주변조직 사이에 지방소거가 뚜렷하게 잘 되어진 것 을 육안으로 확인 할 수가 있었다. 췌장벽(pancreas wall) 에 대한 묘출도는 TSE-SPIR(1.90 \pm 0.2$)$, GE-PROSET $(2.80 \pm 0.6)$ 로 GE-PROSET가 높게 나타났으며 $(\mathrm{p}=0.00004)$, 그림 2에서 보여 주고 있다. 그림 2에서 $\mathrm{b}$ 그림 (GE-PROSET)이 a 그림(TSE-SPIR)에 비해 화살표를 보 았을 때 췌장과 주변조직에 차이가 크게 나며, 췌장의 윤 관이 뚜렷한 것을 알 수가 있었다. 복부 검사 시 GE-PROSET 검사가 췌장질환 진단에 유용한 기법이라는 것을 알 수 있었다. 전반적으로 그림 1,2 를 비교해 보았 을 때 두 기법 모두 지방소거는 되었지만, 특히 GE-PROSET 기법은 자장의 불균일성으로 인하여 대둔근 
부분(gluteus maximus, arrow head)을 비롯하여 전체적으 로 균일하게 지방소거가 이루어지지 않았고, 이러한 원인 은 자장의 불균일성으로 인하여 주파수의 이동이 발생하 여 지방소거가 아닌 물 소거(water suppression) 현상이 나타난 것을 알 수가 있었다(Fig. 1 b, arrow). 두 기법에 대한 이러한 결과들은 지방소거에 관점을 두었을 때 TSE-SPIR 기법이 높은 정보를 제공하게 되었다.

[Table 1] Quantitative values for fat suppression, Qualitative values for delineation of bowel wall and pancreas

\begin{tabular}{lccc}
\hline & ${ }^{\mathrm{a})}$ TSE-SPIR & ${ }^{\text {b) }}$ GE-PROSET & $p$ \\
\hline${ }^{\mathrm{c})} \mathrm{SNR}_{1}$ & $31.50 \pm 05.98$ & $4.15 \pm 00.98$ & 0.00021 \\
${ }^{\mathrm{d})} \mathrm{SNR}_{2}$ & $32.39 \pm 06.01$ & $7.03 \pm 0.2 .07$ & 0.00085 \\
${ }^{\mathrm{e})} \mathrm{CNR}_{1}$ & $152.69 \pm 15.87$ & $74.54 \pm 10.14$ & 0.00074 \\
${ }^{\mathrm{f})} \mathrm{CNR}_{2}$ & $126.12 \pm 11.19$ & $68.78 \pm 09.26$ & 0.00002 \\
${ }^{\mathrm{g})} \mathrm{BWD}$ & $2.40 \pm 0.4$ & $1.80 \pm 00.30$ & 0.00027 \\
${ }^{\mathrm{h})} \mathrm{PD}$ & $1.90 \pm 0.2$ & $2.80 \pm 00.60$ & 0.00004 \\
\hline
\end{tabular}

Note - Data are given as mean \pm standard. deviation.

${ }^{a)}$ TSE-SPIR : turbo spin echo-spectral presaturation inversion

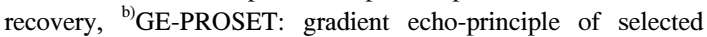
excitation technique. ${ }^{\text {c) }}$ SNR1 : signal to noise ratio in retroperitoneal fat. ${ }^{\mathrm{d})} \mathrm{SNR} 2$ : signal to noise ratio in mesenteric fat. ${ }^{\text {e) }} \mathrm{CNR} 1$ : contrast to noise ratio in retroperitoneal fat. ${ }^{\text {f) }} \mathrm{CNR} 2$ : contrast to noise ratio in mesenteric fat. ${ }^{\mathrm{g}} \mathrm{BWD}$ : bowel wall delineation. ${ }^{\text {h) }} \mathrm{PD}$ : pancreas delineation.
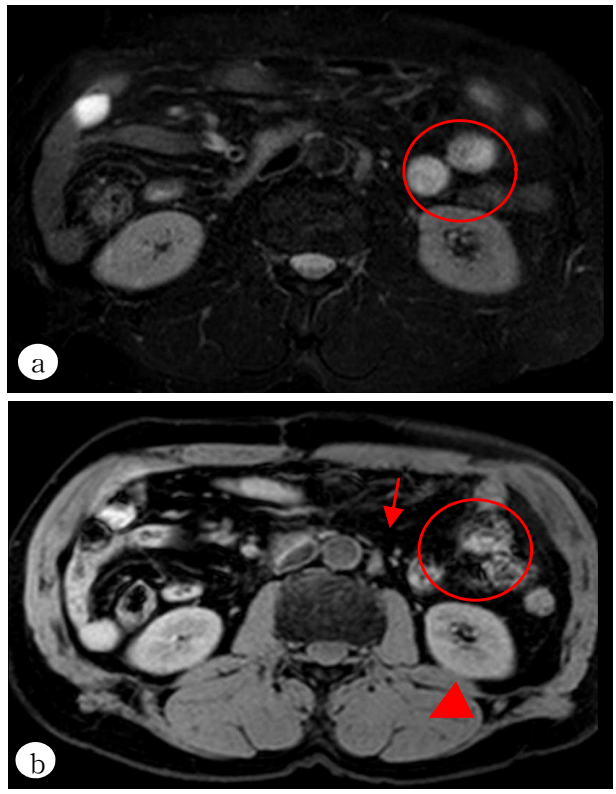

[Fig. 1] 42-year-old(female) normal volunteer. Bowel wall margins(circle) was well demonstrated on TSE-SPIR(a) than on GE-PROSET images(b).
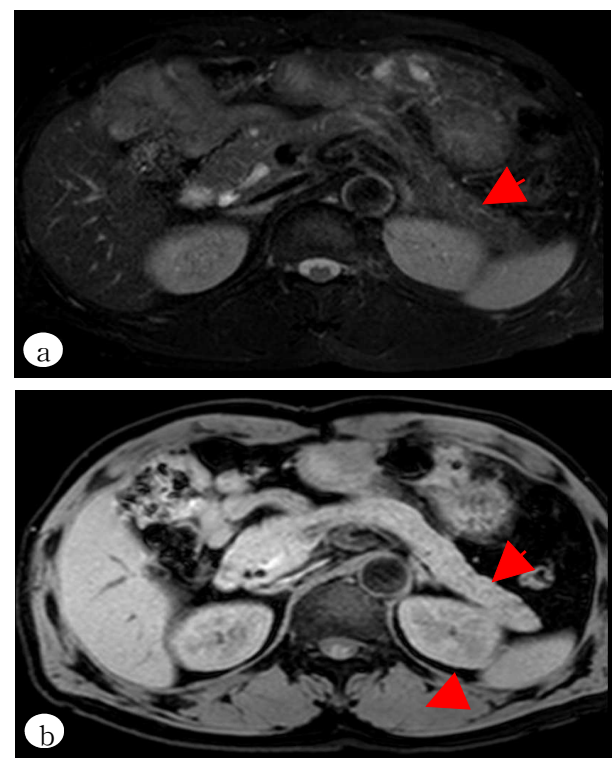

[Fig. 2] 50-year-old(male) normal volunteer. Pancreas wall margins(arrow) was well demonstrated on GE-PROSET(b) than on TSE-SPIR images(a).

\section{4. 고찰}

본 실험을 통하여 임상적 복부 영상에 관한 두 기법에 대한 이점에 대하여 평가를 할 수가 있었고, 후복막 지방 과 장간막 지방에 대한 신호대 잡음비와 대조도대 잡음 비를 측정하여 지방소거 정도를 평가할 수가 있었다. 현 재 임상에서는 자기공명영상기기를 이용하여 복부 질환 평가에 있어서 지방을 소거하는 방법으로 STIR(Short TI Inversion Recovery), SPIR(Spectral Presaturaion with Inversion Recovery, SPAIR(Spectral Attenuated Inversion Recovery), PROSET(PRinciple Of Selected Excitation Technique) 기법이 주로 사용되고 있다[14-16]. 이들 기법 들은 이론적 장, 단점에 따라 복부 및 사지 질환검사에 적절하게 사용을 하고 있다. 본 평가에서는 TSE는 SPIR, $\mathrm{GE}$ 는 PROSET 방법을 적용하여 실험을 하게 되었다. SPIR은 일반적으로 사용되는 CHESS 기법에 STIR 기법 을 접목시킨 것으로 STIR의 반전 RF 펄스로 지방성분만 반전시킨 후 IR(inversion recovery) 동안 지방 신호가 null point(o)가 될 때 여기 RF 펄스를 인가해서 지방을 소거하는 방법이다[Fig. 3]. 


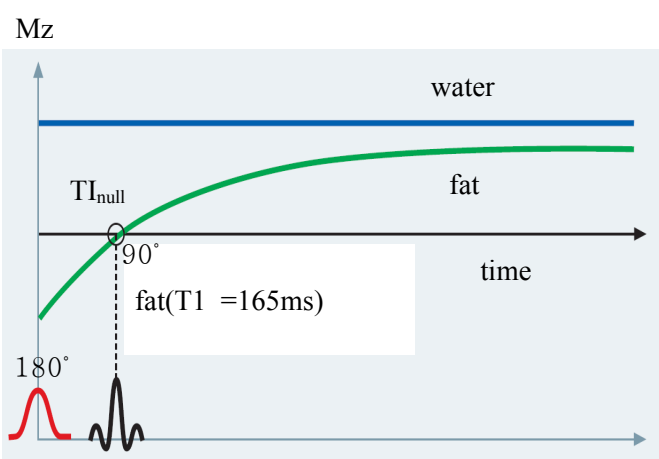

fat

[Fig. 3] SPIR pulse diagram for fat suppression

IR 값에 따라 지방소거 정도가 차이기 있기 때문에 본 실험에서는 기본보고에 제시되었던 IR 값 $165 \mathrm{~ms}$ 주어서 지방 신호가 TInull point(o)가 될 때 RF 펄스를 인가하여 지방을 소거하도록 하였다[17]. SPIR 방법은 자장의 균일 성에 민감하게 영향을 미치기 때문에 $\mathrm{FOV}$ (field of view) 를 $300 \mathrm{~mm}$ 로 최대한 작게 선정하여 자장의 균일성을 유 지하도록 하였다. TSE 펄스시퀀스는 turbo factor(TF)에 의한 대조도를 크게 좌우하기 때문에 강한 $\mathrm{T} 2$ 강조영상 지방소거를 얻기 위해 본 실험에서는 $\mathrm{TF}$ 를 12 번을 인가 하도록 하였다. TF가 12 개 이하가 될 때에는 T2 강조영 상 효과가 아닌 양성자 밀도(proton density) 효과를 발생 할 수가 있다. Olivier Hauger 보고에서 TF를 7번 인가해 서 검사했을 때 지방조직 소거에 대한 $\mathrm{SNR}$ 값이 $15.5 \pm$ 4.7 값으로 본 실험의 $\mathrm{SNR}$ 값 $31.50 \pm 05.98$ 에 비해 아 주 낮은 결과 이었다 [18]. 또한 12 번 이상을 인가했을 때에는 검사시간(scan time)은 감소하나 여러 번의 $180^{\circ}$ 인가로 인하여 영상의 흔들림(blurring)이 발생 할 수가 있기 때문이다. 이 기법 또한 $\mathrm{TR}$ 과 $\mathrm{TE}$ 값을 조정하고, ETL(echo train length)를 조정하여 조영제 주입 후 T1강 조영상 지방소거를 획득할 수 있는 기법이기도 한다. 기 존보고에는 조영제 주입 후 THRIVE 펄스시퀀스를 이용 한 SPIR 방법으로 복부 질환 중 간전이(liver metastasis), 간암(hepatocelullar carcinoma), 전립선암(prostate cancer) 등을 평가하고 있다[19-21]. GE 에서는 PROSET 방법을 적용하였다. 이 방법은 먼저, $22.5^{\circ}$ 여기 펄스를 인간한 후 지방(black color)과 물(red color)은 동위상(in-phase) 상태에 놓이게 된다.

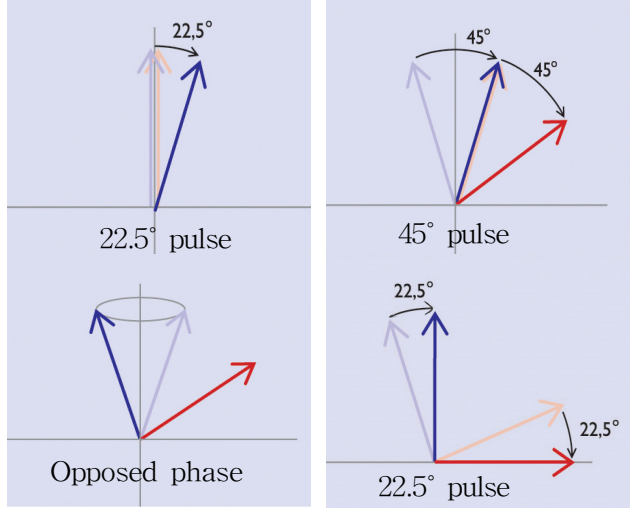

[Fig. 4] PROSET pulse diagram for fat suppression.

이후 일정한 시간이 지난 후 두 조직은 작은 주파수 차이로 인하여 탈위상(opposite phase)을 형상하게 되었 을 때 $45^{\circ}$ 여기 펄스를 인가하여 두 조직이 다시 탈위상 될 때 까지 기다린다. 이후 마지막으로 $22.5^{\circ}$ 여기 펄스 를 인가하게 되면, 물 조직은 신호를 발생하게 되지만 지 방 조직은 종축성분에서 회전하고 있기 때문에 지방을 신호를 형성하지 않고 제거 되는 원리이다. $180^{\circ}$ 가 아닌 $45^{\circ}$ 이하로 여기 펄스를 적용하기 때문에 SPIR 기법에 비 해 검사시간이 짧다는 장점을 가지고 있다[Fig. 4].

기존 보고에서 $\mathrm{GE}$ 지방소거기법은 HASTE 지방소거 기법에 비하여 정성적 분석에서 $96 \%$ 와 $60 \%$ 로 HASTE 지방소거 기법에 비하여 $\mathrm{GE}$ 지방소거기법이 높은 평가 를 받았다[22]. 그러나 본 실험에서 TSE 지방소거 기법과 $\mathrm{GE}$ 지방소거 기법을 비교했을 때 정량적, 정성적 평가결 과가 $83.3 \%$ 와 $13.3 \%$ 로 췌장묘출을 제외하고 모두 TSE 지방소거 기법이 우위의 결과를 얻었다(Table $1, \mathrm{p}<$ 0.05). GE-PROSET 기법은 TR을 짧게 하여 지방소거를 할 수가 있는 장점이 있기 때문에 $204 \mathrm{~ms}$ 로 최대한 작게 설정하여 실험을 하였다. 또한 숙임각(flip angle)을 $25^{\circ}$ 이상 값을 변환 시켰을 때는 $\mathrm{T} 1$ 강조영상 지방소거까지 가능한 기법이다. 실제 임상에서는 복부영역 검사에서 GE-PROSET 기법은 T2, T1 지방소거로 병리학적 정보 및 해부학적 정보를 제공하는데 주로 사용하고 있으나 조영제 주입 후 동적인 영상에는 적용하고 있지 않다. 그 이유는 조영제 주입 후 GE-PROSET 기법은 자기감수성 (magnetic susceptibility)효과로 인하여 SNR, CNR이 많 이 떨어지므로 혈류 역학적 정보를 얻는 데는 큰 도움이 되지 않고 있다[21]. 그 외에 지방(fat)과 장(bowel)영역에 가스가 차있어 자화전이효과(effects of magnetization transfer)로 인하여 영상에 왜곡이 심하기 발생하기도 한 다[23]. GE-PROSET 기법에서 가장 큰 장점은 인체의 물 
(water) 성분을 기준으로 지방소거를 하기 때문에 연골 (cartilage)[24] 및 탈장(herniations), 액체(fluid)[25] 성분 을 묘출하는데 최적의 펄스시퀀스이고, 복부영역에서는 동맥류(aortic aneurysms)[26]을 비롯하여 지방간[27] 평 가에 유용하게 사용되고 있다. 특히 지방이 많이 분포되 어 있는 여성 유방평가에도 기존 보고에 의하면 많이 적 용되고 있다[28]. 이러한 장점은 복부 장기를 묘출하는데 있어서 장기경계를 뚜렷하게 묘출된다는 점이다. 이러한 특성은 본 실험에서 나타난 것과 같이 GE-PROSET 기법 이 췌장을 비롯하여 주변 장기가 잘 묘출된 점이다. 본 실험에서 제한점이 있다면 첫째, 다양한 질환을 대상으로 평가를 하지 않는 점, 그러나 지방소거에만 중점을 갖는 다면 큰 차이가 없을 거라고 생각한다. 둘째, 정확한 지방 소거를 위해 여기(exciting) RF 펄스를 인가하기 전에 체 적(volume) shimming을 하지 않았다는 점, 두 기법 모두 자동(auto) shimming을 했기 때문에 정량적으로 지방소 거를 평가하는데 큰 차이가 없을 것이라고 사료된다.

\section{5. 결론}

결론적으로, $\mathrm{T} 2$ 강조 지방소거 복부자기공명영상에서 GE-PROSET 지방소거기법 보다 TSE-SPIR 지방소거기 법이 전반적으로 지방소거가 잘되어 우위성 있은 평가를 얻었다. 특히 TSE-SPIR 기법은 장 주변에 가스가 분포되 어 있는데도 불구하고, 자장의 균일성을 유지하면서 지방 이 잘 제거 되었다. GE-PROSET 지방소거 기법은 TSE-SPIR 기법보다 낮은 평가를 얻었지만, 복부 내 췌장 을 비롯하여 주변장기를 묘출하는데 도움이 되었으므로 복부 질환에 대한 임상적 가치로 유용한 기법이라고 생 각한다. 이에 본 저자는 두 기법에 대한 장, 단점을 고려 하여 볼 때 복부질환에 대한 지방소거 검사 시 두 기법 에 대한 장점을 적용하여 적절하게 사용하기를 권장한다.

\section{References}

[1] T.W. Chan, J. Listerud, H. Y. Kressel, "Combined chemical-shift and phase-selective imaging for fat suppression: theory and initial clinical experience", Radiology, Vol. 181, No. 1, pp. 41-47, 1991.

[2] K. Darge, S. A. Anupindi, D. Jaramillo, " MR Imaging of the Abdomen and Pelvis in Infants, Children, and Adolescents" Radiolgy, Vol. 261, No. 1, pp. 12-29, 2011.
[3] A. M. Naraghi, L. M. White, C. Patel, G. Tomlinson, E. C. Keystone, " Comparison of 1.0-T Extremity MR and 1.5-T Conventional High-Field-Strength MR in Patients with Rheumatoid Arthritis", Radiology, Vol. 251, No. 3, pp. 829-837, 2009.

DOI: http://dx.doi.org/10.1148/radiol.2521081507

[4] A. Oto, R. D. Ernst, R. Shah, M. Koroglu, G. Chaljub, A. F. Gei, N. Zacharias, G. Saade, " Right-lower-quadrant pain and suspected appendicitis in pregnant women: evaluation with MR imaging. initial experience", Radiology Vol. 234, No. 2, pp. 445-451, 2005. DOI: http://dx.doi.org/10.1148/radiol.2341032002

[5] F. Maccioni, A. Bruni, A. Viscido, M. C. Colaiacomo, A. Cocco, C. Montesani, R. Caprilli, M. Marini, "MR imaging in patients with Crohn disease: value of T2versus T1-weighted gadolinium-enhanced MR sequences with use of an oral superparamagnetic contrast agent", Radiology, Vol. 238, No. 2, pp. 517-530, 2006. DOI: http://dx.doi.org/10.1148/radiol.2381040244

[6] J. Florie, M. N. Wasser, K. Arts-Cieslik, E. M. Akkerman, P. D. Siersema, J. Stoker, "Dynamic contrast-enhanced MRI of the bowel wall for assessment of disease activity in Crohn's disease", AJR Am J Roentgenol, Vol. 186, No. 5, pp. 1384-1392, 2006.

DOI: http://dx.doi.org/10.2214/AJR.04.1454

[7] M. M. von Falkenhausen, G. Lutterbey, N. Morakkabati -Spitz, O. Walter, J. Gieseke, R. Blomer, W. A. Willinek, H. H. Schild, C. K. Kuhl, "High-Field-Strength MR Imaging of the Liver at $3.0 \mathrm{~T}$ : Intraindividual Comparative Study with MR Imaging at $1.5 \mathrm{~T}$ ", Radiology, Vol. 241, No. 1, pp. 156-166, 2006. DOI: http://dx.doi.org/10.1148/radiol.2411051221

[8] Y. Hirokawa, H. Isoda, Y. S. Maetani, S. Arizono, K. Shimada, T. Okada, T. Shibata, K. Togashi, "Hepatic Lesions: Improved Image Quality and Detection with the Periodically Rotated Overlapping Parallel Lines with Enhanced Reconstruction Technique-Evaluation of SPIO-enhanced T2-weighted MR Images", Radiology, Vol. 251, No. 2, pp. 388-397, 2009.

DOI: http://dx.doi.org/10.1148/radiol.2512081360

[9] J. Augui, O. Vignaux, C. Argaud, J. Coste, H. Gouya, P. Legmann, "Liver: T2-weighted MR Imaging with Breath-hold Fast-Recovery Optimized Fast Spin-Echo Compared with Breath-hold Half-Fourier and Non-Breath-hold Respiratory-triggered Fast Spin-Echo Pulse Sequences", Radiology, Vol. 223, No. 3, pp. 853-859, 2002. 
DOI: http://dx.doi.org/10.1148/radiol.2233011011

[10] T. Yokoo, M. Bydder, G. Hamilton, M. S. Middleton, A. C. Gamst, T. Wolfson, T. Hassanein, H. M. Patton, J. E. Lavine, J. B. Schwimmer, C. B. Sirlin, " Nonalcoholic Fatty Liver Disease: Diagnostic and Fat-Grading Accuracy of Low-Flip-Angle Multiecho Gradient-Recalled-Echo MR Imaging at 1.5 T", Radiology, Vol. 251, No. 1, pp. 67-76, 2009. DOI: http://dx.doi.org/10.1148/radiol.2511080666

[11] C. Muhle, J. Metzner, G. Brinkmann, B. Kuhn, L. Bischoff, A. Hutzelmann, F. Wesner, M. Heller, "Comparison of T2-weighted turbo spin-echo and ultrafast HASTE sequence in the diagnosis of cervical myelopathies and spinal stenoses and static and kinematic MRT of the spine", Rofo., Vol. 167, No. 5, pp. 467-73, 1997.

DOI: http://dx.doi.org/10.1055/s-2007-1015565

[12] E. L. Hahn , "Spin Echoes", Physics Review. Vol. 80, pp. 580-594, 1950.

DOI: http://dx.doi.org/10.1103/PhysRev.80.580

[13] T. C. Lauenstein, P. Sharma, T. Hughes, K. Heberlein, D. Tudorascu, D. R. Martin, " Evaluation of Optimized Inversion-Recovery Fat-Suppression Techniques for T2-Weighted Abdominal MR Imaging", Journal of Magnetic Resonance Imaging, Vol. 27, No. 6, pp. 1448-1454, 2008.

DOI: http://dx.doi.org/10.1002/jmri.21350

[14] M. R. Schmid, J. Hodler, P. Vienne, C. A. Binkert, M. Zanetti, "Bone Marrow Abnormalities of Foot and Ankle: STIR versus T1-weighted Contrast-enhanced Fat-suppressed Spin-Echo MR Imaging”, Radiology, Vol. 224, No. 2, pp.463-469, 2002,

DOI: http://dx.doi.org/10.1148/radiol.2242011252

[15] M. A. Brown, R. C. Semelka, "MR Imaging Abbreviations, Definitions, and Descriptions: A Review", Radiology, Vol. 213, No. 3, pp. 647-662, 1999.

[16] G. M. Kukuk, J. Gieseke, S. Weber, D. R. Hadizadeh, M. Nelles, F. Traber, H. H Schild, W. A. Willinek, " Focal Liver Lesions at 3.0 T: Lesion Detectability and Image Quality with T2-weighted Imaging by Using Conventional and Dual-Source Parallel Radiofrequency Transmission", Radiolgoy, Vol. 259, No. 2, pp. 421-428, 2011

DOI: http://dx.doi.org/10.1148/radiol.11101429

[17] E. M. Hecht, A. E. Holland, G. M. Israel, W. Y. Hahn, D. C. Kim, A. B. West, J. S. Babb, B. Taouli, V. S. Lee, G. A. Krinsky, " Hepatocellular Carcinoma in the Cirrhotic Liver: Gadolinium-enhanced 3D T1-weighted
MR Imaging as a Stand-alone Sequence for Diagnosis", Radiology, Vol. 239, No. 2, pp. 438-447, 2006. DOI: http://dx.doi.org/10.1148/radiol.2392050551

[18] O. Hauger, E. Dumont, J. F. Chateil, M. Moinard, F. Diard, " Water Excitation as an Alternative to Fat Saturation in MR Imaging: Preliminary Results in Musculoskeletal Imaging", Radiology, Vol. 2002, No.3, pp.657-663, 2002.

DOI: http://dx.doi.org/10.1148/radiol.2243011227

[19] B. Turkbey, P. A. Pinto, H. Mani, M. Bernardo, Y. Pang, Y. L. McKinney, K. Khurana, G. C. Ravizzini, P. S. Albert, M. J. Merino, P. L. Choyke, " Prostate Cancer: Value of Multiparametric MR Imaging at 3T for Detection?Histopathologifc Correlation", Radiology, Vol. 225, No. 1, pp. 89-99, 2010.

DOI: http://dx.doi.org/10.1148/radiol.09090475

[20] Y. K. Kim, W. J. Lee, M. J. Park, S. H. Kim, H. Rhim, and D. G. Choi, " Hypovascular Hypointense Nodules on Hepatobiliary Phase Gadoxetic Acid-enhanced MR Images in Patients with Cirrhosis: Potential of DW Imaging in Predicting Progression to Hypervascular HCC", Radiology, Vol. 265, No. 1, pp. 104-114, 2012. DOI: http://dx.doi.org/10.1148/radiol.12112649

[21] M. W. Wilson, R. K. Kerlan, N. A. Fidelman, A. P. Venook, M. L. Berge, J. Koda, R. L. Gordon, “ Hepatocellular Carcinoma: Regional Therapy with a Magnetic Targeted Carrier Bound to Doxorubicin in a Dual MR Imaging/ Conventional Angiography Suite-Initial Experience with Four Patients", Radiology, Vol. 230, No. 1, pp. 287-293, 2004.

DOI: http://dx.doi.org/10.1148/radiol.2301021493

[22] R. N. Low, C. P. Sebrechts, D. A. Politoske, M. T. Bennett, S. Flores, R. J. Snyder, J. H. Pressman, "Crohn Disease with Endoscopic Correlation: Single-Shot Fast Spin-Echo and Gadolinium-enhanced Fat-suppressed Spoiled Gradient-Echo MR Imaging", Rdadiology, Vol. 222, No. 3, pp. 652 - 660, 2002.

[23] A. B. Rosenkrantz, P. Storey, A. G. Gilet, B. E. Niver, J. S. Babb, C. H. Hajdu, V. S. Lee, " Magnetization Transfer Contrast-prepared MR Imaging of the Liver: Inability to Distinguish Healthy from Cirrhotic Liver", Radiology, Vol. 262, No. 1, pp.136-143, 2012. DOI: http://dx.doi.org/10.1148/radiol.11111043

[24] M. W. Ahn, U. S. Kim, G. B. Kim," Usefulness of the ProSet MRI for Diagnosis of the Extraforaminal Disc Herniation", J Korean Soc Spine Surg., Vol. 20, No. 1, pp. 28-33, 2013.

DOI: http://dx.doi.org/10.4184/jkss.2013.20.1.28 
[25] L. Z. Yi," The MRI PROSET Sequence Imaging and Clinical Application Value on Lumbosacral Nerve Root", Guangxi Medical University, 2009.

[26] Alerci M, Oberson M, Fogliata A, Gallino A, Vock P, Wyttenbach R., "Prospective, intraindividual comparison of MRI versus MDCT for endoleak detection after endovascular repair of abdominal aortic aneurysms", Eur Radiol., Vol. 19, No. 5, pp. 1223-1231, 2009.

DOI: http://dx.doi.org/10.1007/s00330-009-1318-5

[27] N. Arai, T. Miyati, S. Matsunaga, Y. Motono, Y. Ueda, H. Kasai, Y. Suzuki, T. Matsuda, " New Method of Determining Regional Fat Fraction with Modulus and Real Multiple Gradient-echo(MRM-GRE)", Jpn J Radiol Technol., Vol. 63, No. 3, pp. 312-318, 2007.

DOI: http://dx.doi.org/10.6009/jjrt.63.312

[28] P. D. Friedman, S. V. Swaminathan, R. Smith, " SENSE Imaging of the Breast", AJR., Vol. 184, No. 2, pp. 448-451, 2005.

DOI: http://dx.doi.org/10.2214/ajr.184.2.01840448

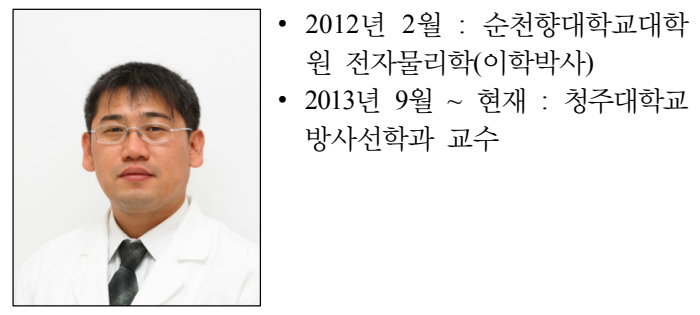

<관심분야>

자기공명영상원리, 방사선 물리 